\title{
Three histopathological types of retinoblastoma and their relation to heredity and age of enucleation
}

Annette C Moll, Jan Willem Koten, Deezi A E Lindenmayer, Linda A Everse, Karel E W P Tan, Alfred Hamburg, Joop A J Faber, Willem Den Otter

\begin{abstract}
The histopathology of 61 eyes was studied with special attention to the morphology of the retina adjacent to the main tumour. Three retinal types were distinguished. Retina type 1 (RT-1, 28 specimens) contained a single tumour that was sharply demarcated from surrounding normal retina. In retina type 2 (RT-2, 29 specimens) large parts of the retina were affected and the main tumour mass gradually blended with the adjacent pathological retina. Retina type 3 (RT-3, four specimens) was characterised by a retina almost entirely affected by diffuse tumour growth.

RT-1 correlated significantly with early enucleation (0-3 years) both in hereditary and non-hereditary cases. RT-2 was seen in eyes enucleated later (2-5 years). The progressing tumour may release growth factors in the intraocular space that stimulate the cells of the adjacent retina and lead to multiple new primary tumours in the adjacent retinal area. RT-3 was only present in non-hereditary cases with late enucleation (at 2-5 years).

Hereditary retinoblastoma cases are usually detected early. Therefore in hereditary cases RT-1 is significantly more common than RT-2. In 25 eyes of the 44 patients with unilateral sporadic retinoblastoma, multifocal tumours of the retina were observed. Such cases should not mistakenly be classified as hereditary cases on the basis of the histological pattern of multifocality of the tumour process. (F Med Genet 1996;33:923-927)
\end{abstract}

Key words: retinoblastoma; histopathology; genetic counselling.

Retinoblastoma is a rare, malignant paediatric tumour of the retina which occurs in a hereditary (30-40\% of the patients) and a nonhereditary form $(60-70 \%) .{ }^{1}$ At least two mutational events are required for development of retinoblastoma, ${ }^{23}$ namely inactivation of both alleles of the retinoblastoma tumour suppressor gene (RB1) on chromosome $13 \mathrm{q} 14 .^{45}$ The hereditary form of retinoblastoma results from a germline mutation in one of the $\mathrm{Rb}$ alleles. This mutation is present in all somatic cells including the retinal cells. Therefore, the development of a retinoblastoma in a person with the inherited form only requires a single additional mutation as opposed to the two mutations required for the development of a non-hereditary retinoblastoma. This results not only in earlier but also in more frequent appearance of tumours, causing the well known multifocality and bilaterality of hereditary retinoblastoma. ${ }^{2}$

Among hereditary cases a familial hereditary form and a sporadic hereditary form can be distinguished. An indication for the familial hereditary form is a parent or a relative with retinoblastoma, implying that one of the parents must carry a mutated RB1 gene. In the sporadic hereditary form, no other family members are affected and the patient is the first person in the family with retinoblastoma. ${ }^{1}$

In the non-hereditary form of retinoblastoma, the RB1 mutation is found exclusively in the tumour cells of the retina, and one eye is unifocally affected. Approximately $10 \%$ of the hereditary retinoblastoma patients are also affected unilaterally, ${ }^{1}$ so hereditary retinoblastoma patients without family history (sporadic hereditary retinoblastoma) may be incorrectly classified as non-hereditary if the tumour occurs unilaterally.

Hereditary retinoblastoma is diagnosed earlier (in disease progression) than non-hereditary retinoblastoma because babies in families where RB is known to occur are checked for retinoblastoma more frequently. ${ }^{6-8}$ Furthermore, hereditary retinoblastoma also occurs earlier than non-hereditary retinoblastoma because of the shortened carcinogenic pathway.

The ability to distinguish between hereditary and non-hereditary retinoblastoma is clinically important as in the former case the second eye may also become affected. Furthermore, in hereditary cases there is an increased risk of second primary tumours. ${ }^{910}$ Together these risks necessitate frequent and thorough screening of hereditary retinoblastoma patients, a costly process which is unnecessary in nonhereditary retinoblastoma patients. Because of this, methods are needed to distinguish between hereditary and non-hereditary retinoblastoma, which can be applied at the time of first tumour occurrence/enucleation. Although it is known that retinoblastoma is caused by a defect in the RB1 gene, the exact defect in this gene can vary or the test is often not informative. ${ }^{112}$ This makes it difficult and time consuming to determine whether a given mutation in a given person is germline (hereditary) or somatic (non-hereditary). ${ }^{13}$ For these reasons genetic screening of all re- 


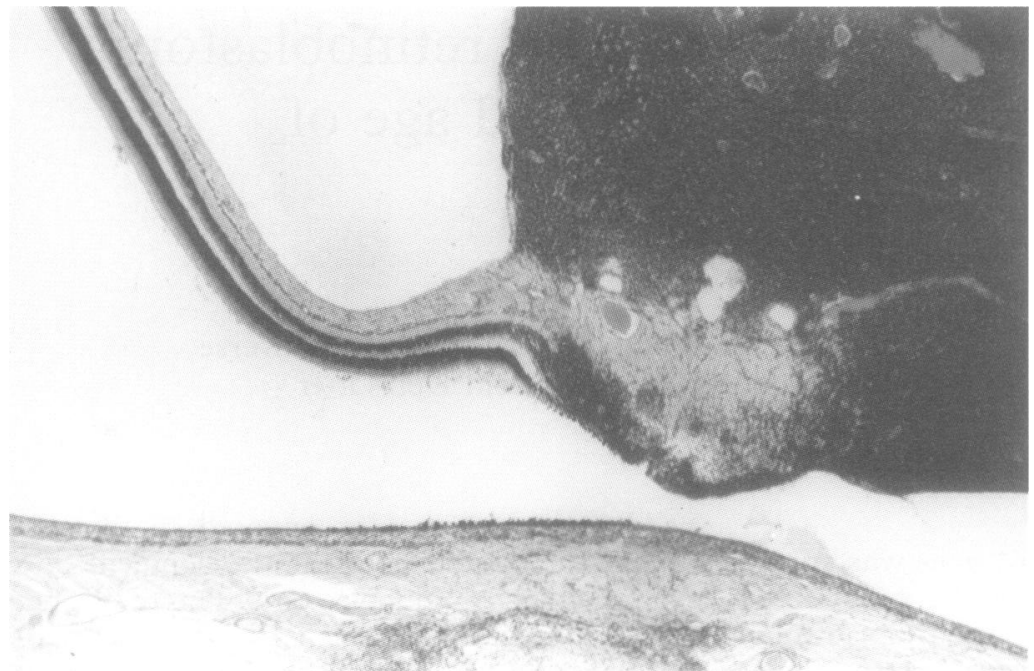

Figure 1 Retina type 1. The retinal cell layers are completely normal. There is an abrupt transition from this normal retina to the tumour tissue.

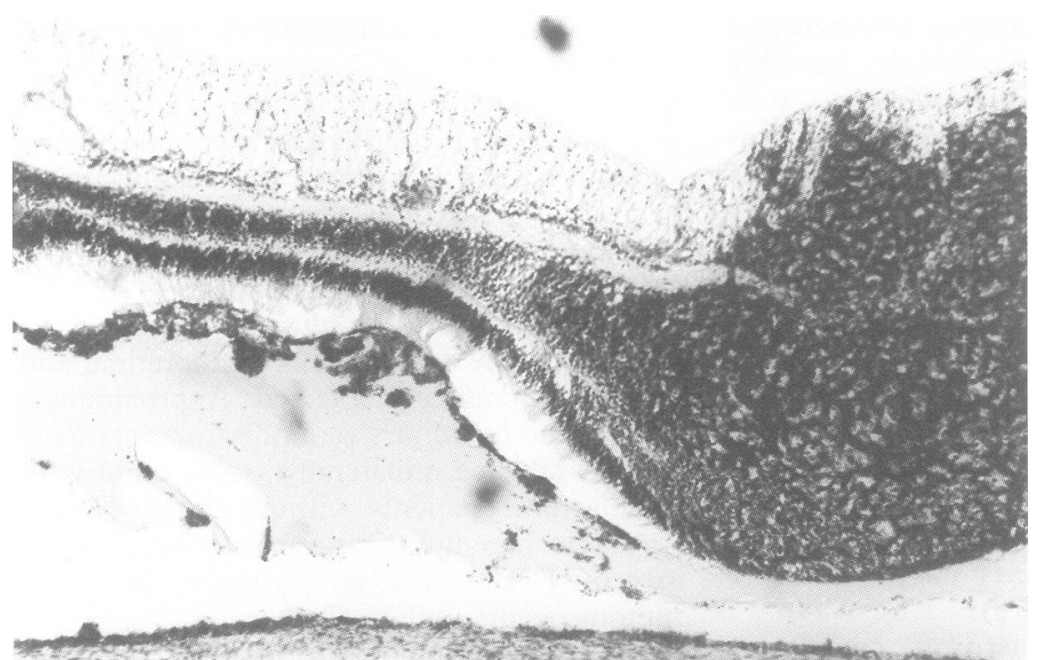

Figure 2 Retina type 2. The whole retina is changed pathologically. There is a widening of the nuclear cell layers. The outer nuclear layer is wider than the inner nuclear layer. The nuclei of the retinal cells are polymorphic. There is a gradual transition from the pathologically affected retina to the tumour.

tinoblastoma patients is not yet a practical means of determining whether or not a given patient has the hereditary or the non-hereditary form of the disease and other means must be sought.

On the basis of these considerations, we have reviewed histological sections of retinoblastoma cases in conjunction with clinical data in order to establish histological criteria for the distinction between hereditary and non-hereditary retinoblastoma. Attention was particularly focused on the retina adjacent to the tumour, since in hereditary cases all retinal cells are affected by an inherited RB1 mutation. As explained above, this may be reflected in multifocal tumour growth.

\section{Materials and methods}

The Dutch Retinoblastoma Register contains virtually all retinoblastoma cases occurring in the Netherlands from 1945 onwards. Histopathological sections of 79 eye specimens (from 77 patients) obtained between 1956 and 1990 were retrieved from this registry. In addition, family history, clinical data, and chromosomal or DNA analysis (if available) of the patients were gathered from the patients' hospital files and the Dutch Retinoblastoma Register.

Retinoblastoma was regarded as hereditary in cases of bilaterality, family history of retinoblastoma, or a defect in the RB1 gene. Only primary enucleated eyes were examined; eyes previously treated with radiotherapy, chemotherapy, cryotherapy, or light coagulation were excluded because treatment can affect the adjacent retina. Sixty one eyes (from 61 patients) fulfilled these criteria.

Sections were stained with haematoxylineosin and classified blindly (without knowledge of heredity) according to the retina classification given in next paragraph. The characteristics of these 61 eyes were tabulated. In the second review the histological conclusions of the first observation were combined with clinical data. The eyes were examined by one person (JWK) and verified by others (ACM, DAEL, AH).

Special attention was paid to the retina adjacent to the main tumour mass, since differences were expected in the retinal histology of hereditary and non-hereditary cases. Three types of retinal lesion were distinguished. Characteristics of the morphology of the various retina types were as follows.

Retina type 1 (RT-1). The retina adjacent to the tumour is normal, the border between the tumour and the normal retinal layer is sharp, and there is no transition of the normal retina into tumour tissue (fig 1). Generally only a single tumour is present, but occasionally an additional tumour may be present.

Retina type 2 (RT-2). Most parts of the retina in RT-2 are irregularly affected, with a varied histopathology ranging from a normal intact retina to a retinal layer completely replaced by tumour tissue. Intermediate changes include hypercellularity of one of the retinal layers with occasional mitotic figures and small localised microtumours particularly located in hypercellular areas. Retinal cells in such hypercellular areas are frequently polymorphic. There is a gradual transition of pathologically affected retina into the main tumour mass. This variety of lesion was regarded as corresponding to different stages of the carcinogenic process (figs 2 and 3 ). The main tumour mass usually shows features of necrosis.

Retina type 3 (RT-3). The entire retina is diffusely and fairly evenly affected by tumour growth, leaving no normal retinal tissue spared. In addition to the retina, the corpus ciliare, the iris, and the anterior segment are also affected.

An important observation is "seedings" (figs 4 and 5). Apparently, seedings are tumour cells detached from the primary tumour, which come free in the vitreous body or in the subretinal exudate and seed elsewhere on the inner or outer surface of the retina where they form a metastasis. ${ }^{14}$ These metastases can be distinguished clearly from tumours arising de novo in the retina. In type 2 retina, the tumour process is similar to a tumour field in which numerous tumours occur independently (for 


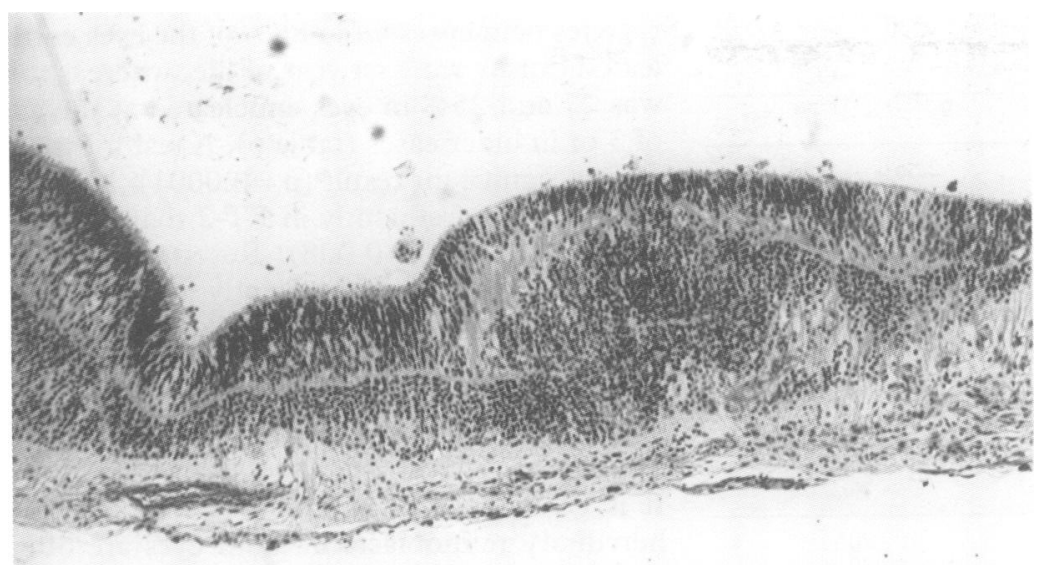

Figure 3 Retina type 2. The whole retina is changed pathologically. There is a widening of the nuclear cell layers. The outer nuclear layer is wider than the inner nuclear layer. The nuclei of the retinal cells are polymorphic. There is a gradual transition from the pathologically affected retina to the tumour.

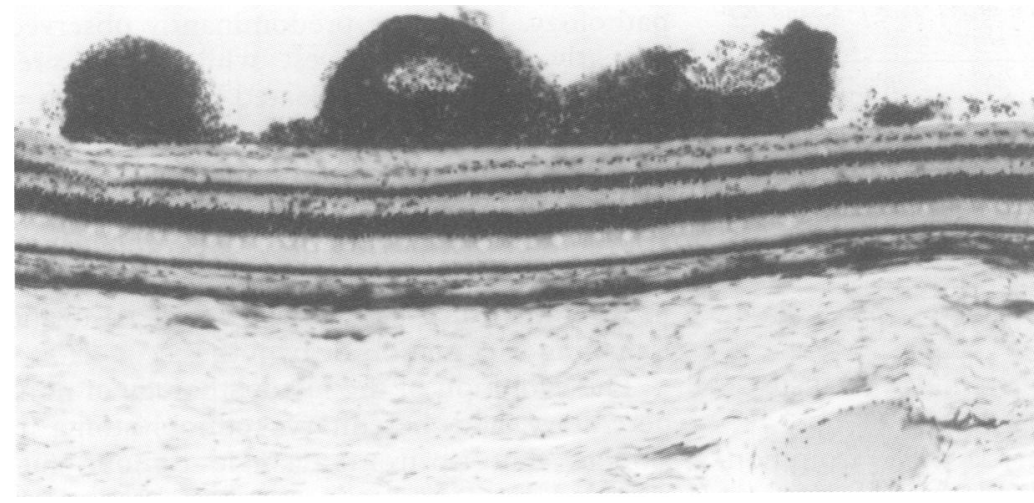

Figure 4 Seeding from a primary retinoblastoma on the retina. Note the abrupt transitions from retina to tumour tissue (courtesy of Professor P van der Valk, Amsterdam).

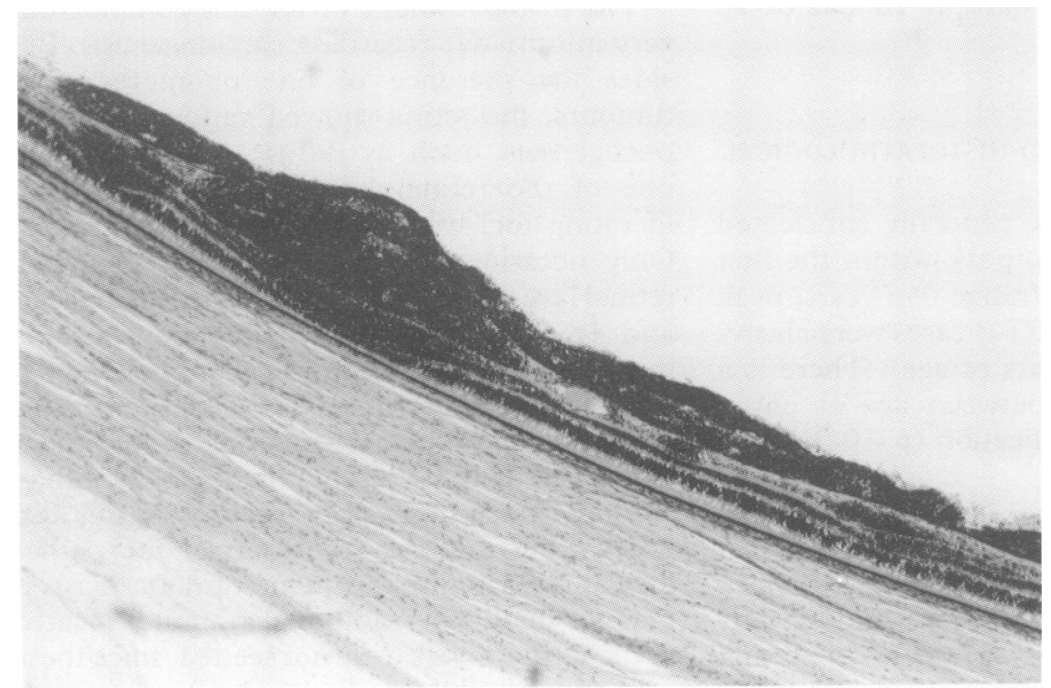

Figure 5 Seeding and retina type 2 (courtesy of Professor P van der Valk, Amsterdam).
Table 1 Clinical data of the retinoblastoma patients

\begin{tabular}{lc}
\hline & No \\
\hline Patients & 61 \\
Affected eyes & 61 \\
Sex & \\
Males & 36 \\
Females & 23 \\
Unknown sex & 2 \\
Unilaterally affected & 44 \\
Bilaterally affected & 16 \\
Unknown laterality & 1 \\
Family history of occurrence & \\
$\quad$ Bilateral & 4 \\
Unilateral & 0 \\
No family history & 57 \\
\hline
\end{tabular}

example, an irradiated skin field in which numerous smaller and larger tumours occur, while in seeding, tumour clusters are present which are isolated and surrounded by ordinary retinal layers.

The degree of differentiation of the tumour was rated "well differentiated" (numerous rosettes), "moderately differentiated" (occasional rosettes), and "poorly differentiated" (no rosettes at all).

Statistical methods used were standard techniques concerning $\mathrm{R} \times \mathrm{C}$ tables. In case of few data exact $p$ values were calculated using the program statXact-Turbo (C Metha, N Patel, CYTEL Software Corporation, Cambridge).

\section{Results}

CLINICOPATHOLOGICAL FINDINGS

The clinical data from the retinoblastoma patients are summarised in table 1 . Cases with a family history of retinoblastoma (four patients) and all bilateral cases ( 16 patients) were classified as hereditary. Unilaterally affected eyes without a family history (44 cases) were considered non-hereditary, though some unilateral cases may be hereditary. In one case the laterality was not recorded.

Table 2 shows that all hereditary cases were enucleated before the age of 2 years. Nonhereditary cases were generally enucleated later (peak at 2 years, range $0-11$ years).

\section{HISTOPATHOLOGICAL CLASSIFICATION AND} HEREDITY

The 61 cases were classified as follows: RT- 1 28 cases, RT- 29 cases, and RT- 3 four cases. Results summarised in table 3 show that the three histological patterns do not provide a definite indication of the hereditary nature of the disease. Retinoblastoma was hereditary in 12 of the 28 cases with RT-1 retinas (43\%) and in four of the 29 patients (14\%) with RT-2 retinas. In hereditary cases RT-1 was significantly more frequent than in non-hereditary cases $(p=0.008)$. We were surprised to find that 25 out of $29(86 \%)$ of the RT-2 retinas, characterised among other things by multiple microtumours (not seeding), belonged to the non-hereditary retinoblastomas. All four RT-3 cases belonged to the non-hereditary group. 
Table 2 Age at enucleation of hereditary and non-hereditary retinoblastoma

\begin{tabular}{|c|c|c|c|c|c|c|}
\hline \multirow[b]{2}{*}{ Age } & \multicolumn{3}{|c|}{ Hereditary } & \multicolumn{3}{|c|}{ Non-hereditary } \\
\hline & No & $\%$ & $A G(\%)$ & No & $\%$ & $A G(\%)$ \\
\hline $\begin{array}{r}0 \\
1 \\
2 \\
3 \\
>4\end{array}$ & $\begin{array}{r}10 \\
4 \\
2\end{array}$ & $\begin{array}{l}62.5 \\
25.0 \\
12.5\end{array}$ & $\begin{array}{l}50.0 \\
33.0 \\
12.5\end{array}$ & $\begin{array}{r}10 \\
8 \\
14 \\
7 \\
5\end{array}$ & $\begin{array}{l}22.6 \\
18.2 \\
31.8 \\
16.0 \\
11.4\end{array}$ & $\begin{array}{c}50 \\
67 \\
87.5 \\
100 \\
100\end{array}$ \\
\hline Total & 16 & 100 & & 44 & 100 & \\
\hline
\end{tabular}

$\mathrm{AG}=$ percentage per age group.

Table 3 Retina type related to heredity

\begin{tabular}{llll}
\hline Retina type & Hereditary & Non-hereditary & Total \\
\hline RT-1 & 12 & 15 & $28^{*}$ \\
RT-2 & 4 & 25 & 29 \\
RT-3 & 0 & 4 & 4 \\
Total & 16 & 44 & 61 \\
\hline
\end{tabular}

*In one case heredity was unknown.

Table 4 Number of tumours in the retina versus heredity

\begin{tabular}{lccc}
\hline No of tumours & Hereditary & Non-hereditary & Total \\
\hline 1 tumour & 6 & 14 & $21^{*}$ \\
2 tumours & 6 & 1 & 7 \\
$>2$ tumours & 4 & 25 & 29 \\
Diffuse & & 4 & 4 \\
Total & 16 & 44 & 61 \\
\hline
\end{tabular}

* In one case heredity was unknown.

Table 5 The age at enucleation of RT-1, RT-2, and RT3

\begin{tabular}{lrrl}
\hline Age & \multicolumn{1}{c}{$R T-1(\%)$} & $R T-2(\%)$ & $R T-3(\%)$ \\
\hline 0 & $17(60.7)$ & $3(10.4)$ & \\
1 & $6(21.4)$ & $7(24.2)$ & $1(25)$ \\
2 & $4(14.3)$ & $11(37.9)$ & $1(25)$ \\
3 & $1(3.6)$ & $5(17.2)$ & $2(50)$ \\
$>4$ & & $3(10.3)$ & $4(100)$ \\
Total & $28(100)$ & $29(100)$ & 4 \\
\hline
\end{tabular}

NUMBER OF TUMOURS IN THE RETINA VERSUS HEREDITY

The majority of the retinas (68.2\%) from nonhereditary cases contained several tumours (table 4). These tumours were not seedings from the primary retinoblastoma, but were intrinsic to the internal or external layer of the retina and appeared to originate from these retinal layers. Retinas with hereditary tumour contained multiple tumours in 10 out of 16 cases $(62.5 \%)$.

AGE AT ENUCLEATION AND HISTOPATHOLOGICAL CLASSIFICATION

RT-1 cases (table 5) are generally enucleated earlier (range 0-3 years, peak within the first year) than type 2 cases (range 0-6 years, peak at 2 years). The eyes of RT- 3 cases were always enucleated late (2-6 years of age). There is a significant correlation between age at enucleation and retina classification $(p=0.016)$.

PRESENCE OF ROSETTES AND AGE OF ENUCLEATION

The presence of rosettes was analysed for correlation with the age of enucleation, the retina type, and heredity. Rosettes were more prominent in early than in late enucleated eyes, rosettes being present in $85 \%$ of the eyes enucleated during the first year of life, whereas this was 27 and $25 \%$ in eyes enucleated at the age of 3 or in older cases (table 6). A test for trend gives a significant result $(p=0.0001)$. Rosettes occurred less frequently in RT-2 than in RT-1 cases (table 7, $\mathrm{p}=0.008$ ). Rosette formation also occurred more frequently in hereditary tumours $(69 \%)$ than in non-hereditary tumours $(40 \%)$.

\section{Discussion}

It is generally accepted that in patients with hereditary retinoblastoma both eyes are often affected with one or more tumours, whereas in non-hereditary retinoblastoma patients only one tumour occurs in one eye. ${ }^{121516}$ This led to our hypothesis that the retina of hereditary retinoblastoma patients will be multifocally affected, whereas the retina of non-hereditary cases would not be affected by new emerging primary tumours besides the main tumour mass. The data collected in this study do not support this hypothesis. This means that these data are very interesting as they reform our thinking on the genesis of retinoblastoma as discussed below.

There appeared to be a significant association between age at enucleation and retinal histopathology. RT-1 was predominantly observed in early enucleated eyes, while RT- 2 predominated in cases enucleated later (exact $\mathrm{p}=$ 0.016). RT-3 was exclusively seen in late cases of (non-hereditary) retinoblastoma. Also tumours with rosettes were more often observed in early tumours than those enucleated later. So rosettes are observed more frequently in RT-1 than in RT-2. These data lead to the following explanation or hypothesis.

The evolution of the histopathology of nonhereditary and hereditary retinoblastoma is similar, that is, from a single tumour surrounded by a normally appearing retina, as observed in many early enucleated cases, to tumoural transformation of the entire retina in late enucleation. This evolution occurs earlier in hereditary tumours than in non-hereditary cases.

The histopathology of RT-2 is complex but very informative regarding carcinogenesis. Besides the presence of one or more larger tumours, the retina showed various stages of oncogenesis, such as diffuse proliferation of one of the retinal layers and discrete proliferating foci with numerous mitotic figures. Only occasional rosettes were observed. All retinal layers (internal nuclear, external nuclear, and even the ganglionic layer) may become involved, probably because all retinal neural elements originate from the same stem cells. The histology shows tumorous involvement as observed in a "tumour field", rather than seeding from a single primary tumour. It is also noteworthy that the transition of the pathological retina into the main tumour mass is gradual in RT-2. Additional tumours located in the retinal layers were not seeded, since these new primary malignancies gradually merge with the adjacent normal retinal cells, but some true 
Table 6 The presence of rosettes in retinoblastoma enucleated at different ages

\begin{tabular}{lrrrrl}
\hline & \multicolumn{2}{l}{ Age $(y)$} & & & \\
\cline { 2 - 6 } Differentiation & 1 & 2 & 3 & $>3$ & All ages \\
\hline Rosettes observed & 17 & 6 & 4 & 2 & 29 \\
No rosettes & 3 & 7 & 12 & 8 & 32 \\
Total & 20 & 13 & 16 & 10 & 61 \\
\hline
\end{tabular}

Table 7 Presence of rosettes in tumour, heredity, and RT types

\begin{tabular}{lrrrl}
\hline Differentiation & \multicolumn{1}{l}{$R T-1$} & $R T-2$ & $R T-3$ & Total \\
\hline Rosettes observed & $19(9)$ & $9(2)$ & $1(0)$ & $29(11)$ \\
No rosettes & $9(3)$ & $20(2)$ & $3(0)$ & $32(5)$ \\
Total & $28(12)$ & $29(4)$ & $4(0)$ & $61(16)$ \\
\hline
\end{tabular}

In brackets the hereditary cases only.

seeding was also observed. Nalbandian and Senft ${ }^{17}$ described similar microneoplasms originating within either the outer or the inner nuclear layers of the retinal tissue outside the retinoblastoma. They did not find a correlation between the microneoplasms and clinical data.

We emphasise that 25 of the 44 eyes of patients with non-hereditary retinoblastoma showed more than two tumours in a single eye. This seems inconsistent with current dogma stressing the unifocality of non-hereditary retinoblastoma. ${ }^{121516} \mathrm{We}$ assume that after a malignant tumour has evolved, it could release growth factors in the isolated and circumscribed intraophthalmic space and affect the adjacent retina by means of paracrine growth stimulation. This might explain the presence of hyperplastic foci in the retinal layers and the emergence of additional primary retinoblastomas in such areas. Thus, more than one tumour could develop in the retina of a non-hereditary retinoblastoma patient if the tumour progresses. Consequently non-hereditary retinoblastoma does not imply a unifocal tumour, as unilateral multifocal retinal tumours frequently occur.

RT-3, the diffuse infiltrating type, was only observed four times in 61 patients. Descriptions of this type have been published previously. ${ }^{18}$ All reported cases of diffuse infiltrating retinoblastoma have been unilateral and sporadic with a negative family history, similar to the four cases we describe. ${ }^{19}$ RT- 3 might thus be RT-2 tumours that are enucleated at a very late stage, where the entire retina is affected.
In conclusion, the histopathology of retinoblastoma cannot provide information about the hereditary origin of the disease. It seems most likely that the reported histological types represent various phases of progression of the tumour process. Furthermore, non-hereditary retinoblastoma does not imply unifocal tumour; multifocal retinal tumours may also occur in non-hereditary cases, particularly if eyes are enucleated late. Such cases should not mistakenly be classified as hereditary cases on the basis of the histological pattern of multifocality of the tumour process. Genetic counsellors should be aware of this phenomenon.

1 Vogel F. Genetics of retinoblastoma. Hum Genet 1979;52: $1-54$.

2 Knudson AG. Mutation and cancer: statistical study of retinoblastoma. Proc Natl Acad Sci USA 1971;68:820-3.

3 DerKinderen DJ. $A$ new concept of oncogenesis with an evaluation in retinoblastoma. Thesis, University of Utrecht, 1987.

4 Cavenee WK, Dryia TP, Philips RA, et al. Expression of recessive alleles by chromosomal mechanism in rerecessive alleles by chromosomal mech

tinoblastoma. Nature 1983;305:779-84.
5 Dryja TP, Rapaport JM, Joyce JM, Petersen RA. Molecular detection of deletions involving band q14 of chromosome 13 in retinoblastoma. Proc Natl Acad Sci USA 1986;83 7391-4

6 Bonaïti-Pellie C, Briard-Guillemot ML, Feingold J, Frezal $\mathrm{J}$. Associated congenital malformations in retinoblastoma Clin Genet 1975;7:37-9.

7 Matsunaga E. Hereditary retinoblastoma: penetrance, expressivity and age of onset. Hum Genet 1976;33:1-15.

8 Schipper J. Retinoblastoma. A medical and experimental study. Thesis, University of Utrecht, 1980.

9 DerKinderen DJ, Koten JW, Nagelkerke NJD, Tan KEWP, Beemer FA, Den Otter W. Non-ocular cancer in patients with heritable retinoblastoma and their relatives. Int $f$ with heritable retinoblas

10 Moll AC, Imhof SM, Koten JW, et al. Second primary tumors in patients with hereditary retinoblastoma: a registered-based follow-up study 1945-1994. Int f Cance (in press)

11 Lohmann DR, Brandt B, Hopping W, Passarge E, Horsthemke B. Spectrum of small length germline mutation in the RB1 gene. Hum Mol Genet 1994;3:2187-93.

12 Kato MV, Ishizaki K, Toguchida J, et al. Mutations in the retinoblastoma gene and their expression in somatic and tumor cells of patients with hereditary retinoblastoma. Hum Mutat 1994;3:44-51.

13 Shimizu T, Toguchida J, Kato MV, Kanedo A, Ishizaki K, Sasaki MS. Detection of mutations of the RB1 gene in retinoblastoma patients by using exon-by-exon PCR in retinoblastoma patients by using exon-by-exon

14 Zimmerman LE. Retinoblastoma and retinocytoma. In: Spencer WH, ed. Ophthalmic pathology. 3rd ed. Volume 2. Philadelphia: Saunders, 1985:1300.

15 Abramson DH, Fusco Marks R, Ellsworth RM, Tretter P Kitchin FD. The management of unilateral retinoblastoma without primary enucleation. Arch Ophthalmol 1982;100 1249-52.

16 Kingston J, Hungerford J. Retinoblastoma. In: Plowman PN, Pinkerton CR, eds. Paediatric oncology. London Chapman \& Hall Medical, 1992:268-90.

17 Nalbandian R, Senft SH. Histopathology and growth patterns of novel microneoplasms in eyes with retinoblastoma. Saudi $f$ Ophthalmol 1993;7:11-16.

18 Morgan G. Diffuse infiltrating retinoblastoma. $\mathrm{Br} f \mathrm{Oph}$ thalmol 1971;55:600-6.

19 Shields J, Shields C. Spontaneous pseudohypopyon secondary to diffuse infiltrating retinoblastoma. Arch Ophthalmol 1988;106:1301-2. 